\title{
BMJ Open Study protocol: treatment with caffeine of the very preterm infant in the delivery room: a feasibility study
}

\author{
Carlo Dani (D) , ${ }^{1}$ Alessandra Cecchi, ${ }^{2}$ Giulia Remaschi, ${ }^{2}$ Domenica Mercadante, ${ }^{3,4}$ \\ Giancarlo la Marca, ${ }^{5}$ Luca Boni, ${ }^{6}$ Fabio Mosca ${ }^{4}$
}

To cite: Dani C, Cecchi A, Remaschi G, et al. Study protocol: treatment with caffeine of the very preterm infant in the delivery room: a feasibility study. BMJ Open 2020;10:e040105. doi:10.1136/ bmjopen-2020-040105

- Prepublication history and additional material for this paper is available online. To view these files, please visit the journal online (http://dx.doi.org/10. 1136/bmjopen-2020-040105)

Received 05 May 2020 Revised 06 0ctober 2020 Accepted 07 October 2020

Check for updates

(C) Author(s) (or their employer(s)) 2020. Re-use permitted under CC BY-NC. No commercial re-use. See rights and permissions. Published by BMJ.

For numbered affiliations see end of article.

Correspondence to

Dr Carlo Dani; cdani@unifi.it

\section{ABSTRACT}

Introduction Early treatment with caffeine in the delivery room has been proposed to decrease the need for mechanical ventilation (MV) by limiting episodes of apnoea and improving respiratory mechanics in preterm infants. Thus, the purpose of this feasibility study is to verify the hypothesis that intravenous or enteral administration of caffeine can be performed in the preterm infant in the delivery room.

Methods and analysis In this multicentre prospective study, infants with $25^{+0}-29^{+6}$ weeks of gestational age will be enrolled and randomised to receive $20 \mathrm{mg} / \mathrm{kg}$ of caffeine citrate intravenously, via the umbilical vein, or enterally, through an orogastric tube, within $10 \mathrm{~min}$ of birth. Caffeine plasma level will be measured at $60 \pm 15 \mathrm{~min}$ after administration and $60 \pm 15$ min before the next dose $(5 \mathrm{mg} / \mathrm{kg})$. The primary endpoint will be evaluation of the success rate of intravenous and enteral administration of caffeine in the delivery room. Secondary endpoints will be the comparison of success rate of intravenous versus oral administration and the evaluation of the need for MV in treated infants. In the absence of previous references, we arbitrarily decided to study 20 infants treated with intravenous caffeine and 20 infants treated with enteral caffeine. Primary endpoint will be evaluated measuring the success rate of intravenous and enteral caffeine administration which will be considered a success when it is followed by the achievement of the caffeine therapeutic level $(8-25 \mu \mathrm{g} / \mathrm{mL}) 60 \pm 15 \mathrm{~min}$ before administration of the second dose.

Ethics and dissemination The study has been approved by the Italian Medicines Agency (AIFA: AIFA/RSC/P/32755) and by Comitato Etico Pediatrico Regione Toscana. The results will be published in peer-reviewed academic journals.

Trial registration number ClinicalTrials.gov identifier NCT04044976; EudraCT number 2018-003626-91.

\section{INTRODUCTION}

Mechanical ventilation (MV) is one of the most important risk factors for the development of bronchopulmonary dysplasia (BPD) in the preterm infant, due to the early pulmonary inflammation from volume trauma and barotrauma, and the high risk of ventilator-associated pneumonia. ${ }^{1}$ Therefore, in recent years, particular attention

\section{Strengths and limitations of this study}

This is the first study assessing the possibility of giving intravenous or enteral caffeine to preterm infants in the delivery room.

- Administration will be considered a success when it is followed by the achievement of caffeine blood therapeutic level.

- The caffeine plasma level will be measured using the 'dried blood spots'.

- This study is preliminary to a large randomised controlled trial which will assess whether caffeine administered so early can reduce the risk of mechanical ventilation in very preterm infants.

- This research is being conducted across two sites and may not be representative of other neonatal intensive care units.

has been paid to reduce the need for MV and some beneficial interventions, such as early application of nasal continuous positive airway pressure (CPAP) and surfactant treatment, have become widespread. ${ }^{12}$ These have the common objective of promoting the development and maintenance of alveolar functional residual capacity, improving pulmonary compliance, reducing the work of breathing, and favouring gas exchanges. ${ }^{1}{ }^{2}$ Unfortunately, it has been found that nasal CPAP in combination or not with surfactant administration fails to prevent MV in about $45 \%-50 \%$ of treated infants. ${ }^{3}$ In fact, the need for MV often does not depend on the severity of respiratory distress syndrome (RDS), which however remains an important factor, but especially in mild-moderate forms of RDS can be due to the onset of relapsing episodes of apnoea. Therefore, it has been proposed to treat very preterm infants with caffeine in the delivery room already in the first minute of life. In a recent pilot study, Katheria et al randomised 21 infants with gestational age $<29$ weeks to receive $20 \mathrm{mg} / \mathrm{kg}$ of caffeine citrate within 2 hours of life or at 12 hours 
of life. ${ }^{4}$ They found that early treatment decreased the need for MV (27 vs 70\%) in comparison with late treatment and allowed an overall haemodynamic improvement of early treated patients. ${ }^{4}$ Subsequently, Dekker et al randomised 23 infants of 24-29 gestational weeks to receive $10 \mathrm{mg} / \mathrm{kg}$ of caffeine in the delivery room or immediately after arrival in neonatal intensive care unit (4.4 vs 48 min of life). ${ }^{5}$ They found that early treatment significantly increased tidal volume and decreased the need for oxygen therapy in comparison with later treatment. ${ }^{5}$ Although these results were promising, neither study had sufficient statistical power to assess whether early treatment with caffeine is effective in reducing the need for MV in studied infants.

\section{Study hypothesis}

The present feasibility study aims to evaluate the hypothesis that it is operatively possible to administer intravenous or enteral caffeine in the delivery room during infants' postnatal stabilisation when resuscitation may be needed. This study is preliminary to the planning of a subsequent large randomised controlled trial which will assess whether caffeine administered so early can actually reduce the risk of MV in very preterm infants.

\section{Objectives of the study}

\section{Primary objective}

To evaluate the feasibility of administration of intravenous or enteral caffeine in very preterm infants in the delivery room during assistance for their cardiorespiratory stabilisation achieving therapeutic plasma level.
Secondary objectives

To compare the success rate of intravenous versus enteral administration in overall population and to evaluate the need for MV in treated infants.

\section{METHODS AND ANALYSIS \\ Study setting}

This multicentre prospective study will be conducted in two level 3 neonatal intensive care units of the Careggi University Hospital of Florence and Fondazione IRCCS Cà Granda Ospedale Maggiore Policlinico of Milan, after approval by local ethics committees.

\section{Inclusion criteria}

Once the written informed consent of the parents or legal guardians has been obtained (see online supplemental file), inborn infants of $25^{+0}-29^{+6}$ weeks of gestational age at high risk of developing RDS, who do not require MV in the delivery room, will be enrolled in the study.

\section{Exclusion criteria}

Exclusion criteria will be maternal consumption of caffeine before giving birth ( $>2$ cups of coffee in the 6 hours before birth), major congenital malformations, chromosomal syndromes, inherited metabolic disorders and fetal hydrops.

\section{Interventions}

The study design and timeline are summarised in figures 1 and 2. Infants will be electronically randomised to receive $20 \mathrm{mg} / \mathrm{kg} \quad(1 \mathrm{~mL}=20 \mathrm{mg}$ ) of caffeine citrate (Peyona; Chiesi Farmaceutici Spa, Parma, Italy) intravenously, via

\begin{tabular}{|l|l|}
\hline $\begin{array}{l}\text { Inclusion criteria } \\
\begin{array}{l}\text { 1. Inborn infants of } 25^{+0}-29^{+6} \text { weeks of gestational age at high risk } \\
\text { of developing RDS, who do not require MV in the delivery room. }\end{array}\end{array}$ & $\begin{array}{l}\text { Exclusion criteria } \\
\text { 1. Maternal consumption of caffeine before giving birth (>2 cups of } \\
\text { coffee in the } 6 \text { hours before birth); }\end{array}$ \\
$\begin{array}{ll}\text { 2. Parental informed consent } & \begin{array}{l}\text { major congenital malformations, chromosomal syndromes, } \\
\text { inherited metabolic disorders; } \\
\text { 3. fetal hydrops }\end{array}\end{array}$
\end{tabular}

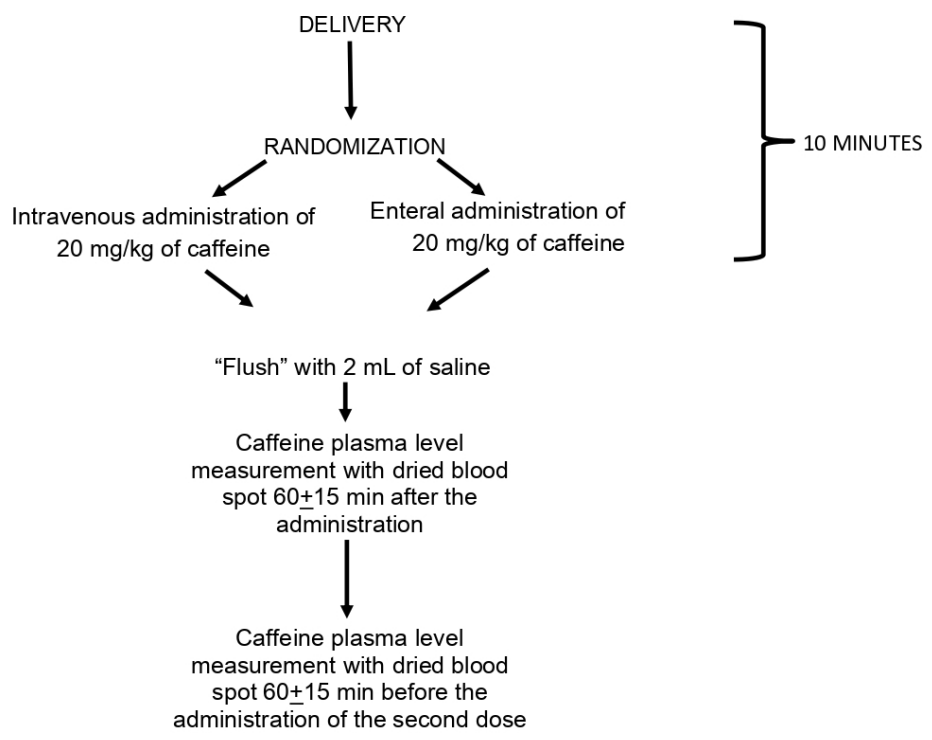

Figure 1 Flowchart of the study. MV, mechanical ventilation; RDS, respiratory distress syndrome. 


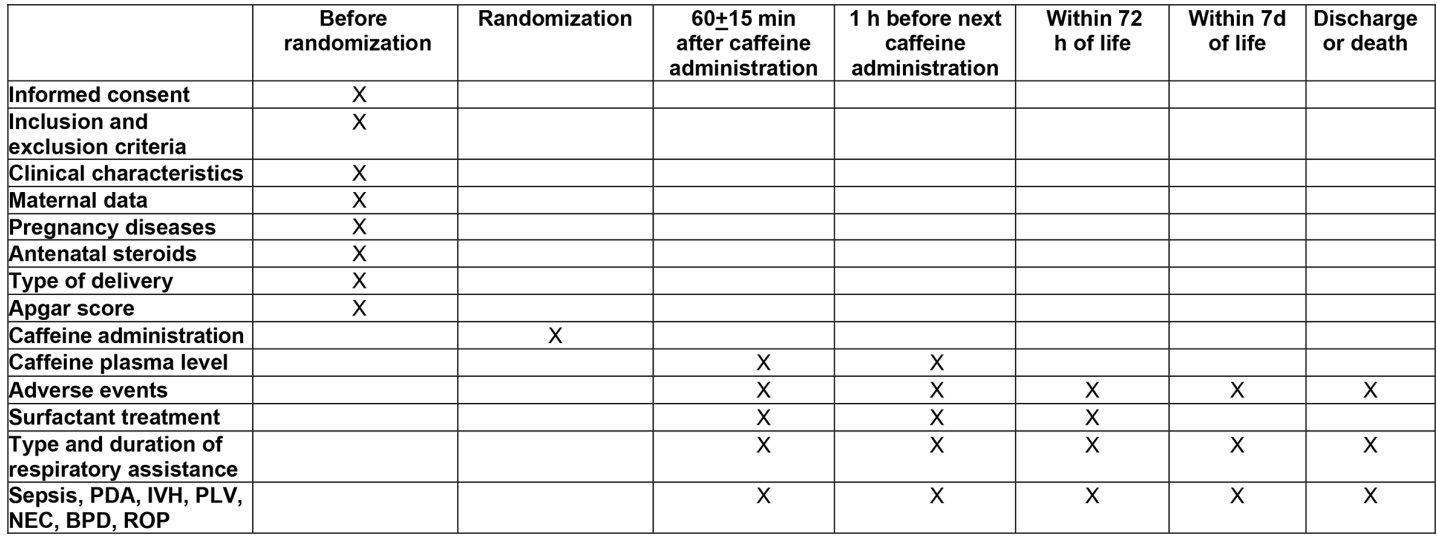

Figure 2 Study timeline. BPD, bronchopulmonary dysplasia; IVH, intraventricular haemorrhage; NEC, necrotizsing enterocolitis; PDA, patency of the ductus arteriosus; PVL, periventricular leukomalacia; ROP, retinopathy of prematurity.

the umbilical vein, or enterally, through an orogastric tube, within $10 \mathrm{~min}$ of birth. The randomisation sequence will be generated from the e-clintrials platform (https:// www.eclintrials.org/ect/O) whose manager is LB.

Intravenous administration will take place via an umbilical venous catheter or a 'butterfly' needle inserted into the umbilical vein. The bolus of caffeine will be followed by the administration of a $2 \mathrm{~mL}$ 'flush' of saline both in the case of administration by venous and enteral route. Completion or failures of administration will be recorded.

The caffeine plasma level will be measured $60 \pm 15 \mathrm{~min}$ after the administration, to evaluate its peak value, and $60 \pm 15 \mathrm{~min}$ before administration of the second dose $(5 \mathrm{mg} / \mathrm{kg} /$ day intravenously) in the neonatal intensive care unit, to evaluate the achievement of therapeutic plasma level $(8-25 \mu \mathrm{g} / \mathrm{mL}) .{ }^{6}$ The plasma level will be measured using the 'dried blood spots' method with spectrometry and 'tandem-mass' liquid chromatography in the Laboratory of Clinical Chemistry and Pharmacology of the A. Meyer Pediatric Hospital of Florence. Blood samples will be collected with heel punctures commonly performed to monitor these patients and stored at $-80^{\circ} \mathrm{C}$ until analysis.

If necessary, resuscitation in the delivery room will be performed following the guidelines of the AAP/AAH. ${ }^{8}$ After admission in the neonatal intensive care unit, infants will be assisted with the following non-invasive respiratory supports: nasal-CPAP, 'bi-level' nasal-CPAP (BiPAP) and nasal intermittent mandatory ventilation (N-IMV). Surfactant (Curosurf, Chiesi, Parma, Italy) will be given $(200 \mathrm{mg} / \mathrm{kg}$ ) according to the InSURE (Intubation-SURfactant-Extubation) or LISA (LessInvasive-Surfactant-Administration) technique in infants requiring $\mathrm{FiO}_{2}>0.30$ to maintain a $\mathrm{SpO}_{2} 90 \%-95 \%$ and in all infants who will need MV.

MV will be started if $\mathrm{pCO}_{2}$ will be $>65 \mathrm{~mm} \mathrm{Hg}$ and $\mathrm{pH}$ $<7.20$, or $\mathrm{pO}_{2}<50 \mathrm{~mm} \mathrm{Hg}$ with $\mathrm{FiO}_{2}>0.50$ after surfactant administration or in case of apnoea ( $>4$ episodes in 1 hour or $>2$ episodes in 1 hour requiring manual ventilation), ${ }^{9}$ and will be continued with the aim of maintaining a $\mathrm{pCO}_{2}$ of $55-65 \mathrm{~mm} \mathrm{Hg}$ and $\mathrm{SpO}_{2}$ of $90 \%-95 \%$, using synchronised MV (patient-triggered ventilation: PTV), volume-controlled MV or high-frequency ventilation (HFV). Patients treated with MV will receive additional doses of surfactant $(100 \mathrm{mg} / \mathrm{kg})$ at the discretion of the attending neonatologist. Patients will be extubated when a good respiratory autonomy is associated with a $\mathrm{FiO}_{2}$ $<0.30$ and a mean airway pressure $<8 \mathrm{cmH}_{2} \mathrm{O}$.

\section{Data collection}

Following data will be recorded for each infant: gestational age; birth weight; birth weight $<10$ th percentile; sex; type of delivery; Apgar score at $5 \mathrm{~min}$; main disorders of pregnancy (pre-eclampsia, premature rupture of membranes, clinical chorioamnionitis, placental abruption); RDS, diagnosis of which will be based on the occurrence of oxygen dependence, tachypnoea, dyspnoea, exclusion of other causes of respiratory failure, and the presence of a typical radiological pattern; treatment with surfactant and nitric oxide; need, type and duration of respiratory assistance (oxygen therapy, NCPAP, BiPAP, N-IMV, PTV, HFV); prenatal and postnatal steroid treatment. In addition, common complications of prematurity will be recorded: patency of the ductus arteriosus requiring pharmacological therapy, necrotising enterocolitis (NEC) <2 grade, BPD, intraventricular haemorrhage $(\mathrm{IVH}) \leq 3$ grade, periventricular leukomalacia (PVL), retinopathy of prematurity (ROP) of grade $>3$ and sepsis. In addition, mortality and the duration of hospitalisation will be reported. The adapted classification of Papile $e t$ $a l$ will be used to classify the severity of $\mathrm{IVH}^{10}$; the diagnosis of PVL will be made in the presence of cystic areas detected by cerebral ultrasound at 40 post-conceptional weeks ${ }^{11}$; ROP will be graded in accordance with the International Classification of $\mathrm{ROP}^{12}$; diagnosis of NEC will be made according to Bell's criteria. ${ }^{13}$ Diagnosis of sepsis will be based on clinical and laboratory data (total neutrophil count, $\mathrm{C}$ reactive protein) confirmed by the presence of at least one positive blood or cerebrospinal fluid culture.

All collected data will be recorded on a web-based electronic case report form, specifically designed for this study. 


\section{Concurrent treatments}

Daily treatment of patients enrolled in the study will be performed according to common practice. In particular, infants with RDS will be treated according to the criteria described in the study design. Infants will receive antibiotic prophylaxis after performance of appropriate diagnostic tests. Antibiotic therapy will be stopped after 3 or 4 days if these tests are negative. Postnatal treatment with steroids may occur in infants with severe respiratory failure during mechanical ventilation and at high risk of mortality.

The enrolled patients can be treated with the following drugs: surfactant, caffeine, doxapram, ibuprofen, paracetamol, indomethacin, dopamine, dobutamine, milrinone, epinephrine, diuretics, antibiotics, glucocorticoids, immunoglobulins, antiepileptics, nitric oxide, analgesics and sedatives.

\section{Adverse effects}

Different adverse effects have been associated to caffeine treatment, although the CAP trial and related studies did not show any significant short-term or long-term adverse effects of caffeine therapy. ${ }^{14}$ We will record the following possible adverse events (AEs): tachycardia, dysrhythmia, gastro-oesophageal reflux and seizures. Diagnosis of gastro-oesophageal reflux will be made on the basis of clinical signs, such as persistent crying, irritability, backarching, feeding and frequent awakening. ${ }^{15}$

The collection of AE data will last until discharge.

\section{Statistical methods}

In the absence of previous studies to use as a reference and as this is a feasibility study, it was arbitrarily decided to study 40 patients, of whom 20 will be treated with intravenous caffeine and 20 with enteral caffeine.

The clinical characteristics of the two groups will be described by calculating their mean values and SD or rates and percentages.

The primary endpoint will be evaluation of the number of infants for whom administration of caffeine intravenously or enterally occurs successfully in the delivery room within $10 \mathrm{~min}$ of life. Administration will be considered a success when it is followed by the achievement of caffeine therapeutic level $(8-25 \mu \mathrm{g} / \mathrm{mL})^{16} 60 \pm 15 \mathrm{~min}$ before the administration of the second dose. We decided to measure caffeine level to have objective evidence that caffeine administration has occurred successfully and that intravenous and enteral administration is equivalent or not.

The secondary objectives will be as follows: comparison of the completion rate of intravenous versus oral administration including infants who do not reach caffeine therapeutic level; comparison of caffeine plasma level obtained with intravenous and enteral administration; evaluation of peak caffeine blood levels; assessment of frequency of MV within the first 72 hours of life in studied infants.

The primary endpoint will be assessed calculating the percentage of cases in which caffeine is successfully administered and therapeutic plasma level reached. Comparisons between infants treated with intravenous or enteral caffeine administration will be performed using the Student t-test for continuous parametric variables, the Wilcoxon rank-sum test for non-parametric continuous variables and the $\chi^{2}$ test for categorical variables. A $\mathrm{p}$ value $<0.05$ will be considered as statistically significant.

\section{Patient and public involvement}

Patients and the public were not (or will not) be involved in the design, or conduct, or reporting, or dissemination plans of this research because in our study we will only anticipate caffeine treatment that will start in the delivery room rather than few hours later in the neonatal intensive care. However, if we will demonstrate that caffeine administration is feasible in the delivery room, we will involve patients and the public in the design, or conduct, or reporting, or dissemination of a subsequent trial to assess whether this strategy can decrease the need of MV in very preterm infants.

\section{ETHICS AND DISSEMINATION}

The study will be carried out in accordance with recognised ethical principles for clinical trials (Helsinki Declaration), with respect for the principles of good clinical practice in the field of clinical investigations on drugs and medical devices and applicable regulations.

The study has been approved by the Italian Medicines Agency (AIFA: AIFA/RSC/P/32755, 31 March 2019). Approval was obtained also from Comitato Etico Pediatrico Regione Toscana for the Careggi University Hospital of Florence, while it is under submission to Comitato Etico Milano Area B for the Fondazione IRCCS Cà Granda Ospedale Maggiore Policlinico of Milan. Parents, relatives and guardians of the enrolled patients will be given all pertinent explanations related to the study and an information sheet about the study will be provided describing the procedure and purpose of the project. Signed parental informed consent is to be obtained by a physician responsible for the study prior to the enrolment. All consents will be obtained before the delivery, possibly at the time of admission of pregnant women at risk of preterm birth.

Every effort will be made to maintain the privacy and confidentiality of patients. To facilitate this, patients' identification data (name, date of birth, medical record number) will be kept in the data set only for the time necessary; therefore, the database will be de-identified and an alphanumeric code will be used. After the data have been entered, patient's sensitive data will be anonymised by assigning a numerical identification code that will only allow identification of the patient to be traced back if necessary.

The principal investigator will be responsible for global monitoring of data and the safety of study participants. The principal investigator will be assisted by other members participating in the study. 
Data will be the property of the promoter and will be shared with the investigators. Ownership of the study data will belong to the investigators involved. The results of the study will be published and may also be the subject of communications, reports or posters at conferences. We declare that the results of the study will be made available for publication.

\section{Current trial status}

Recruitment of participants started in September 2019, and the last participant is expected to reach the primary endpoint in February 2021. Primary data analysis will begin in June 2021.

\section{Ethics approval and consent to participate}

The study has been approved by the Italian Medicines Agency (AIFA: AIFA/RSC/P/32755, march 31, 2019). Approval was obtained also by Comitato Etico Pediatrico Regione Toscana.

\section{Author affiliations}

${ }^{1}$ Department of Neurosciences, Psychology, Drug Research and Child Health, University of Florence, Firenze, Italy

${ }^{2}$ Division of Neonatology, Careggi University Hospital of Florence, Florence, Italy

${ }^{3}$ Fondazione IRCCS Cà Granda Ospedale Maggiore Policlinico of Milan, University of Milan, Florence, Italy

${ }^{4}$ Department of Clinical Sciences and Community Health, Fondazione IRCCS Cà Granda Ospedale Maggiore Policlinico, Milan, Italy

${ }^{5}$ Laboratory of Clinical Chemistry and Pharmacology of the A Meyer Pediatric Hospital of Florence, University of Florence, Florence, Italy

${ }^{6}$ Department of Human Pathology and Oncology, University of Florence, Florence, Italy

Contributors All authors made substantive intellectual contributions to the trial design and manuscript. All revised the manuscript critically. CD and FM conceived of the study. AC, GIM and DM will be responsible for the neonatal care to newborns enrolled. GIM will be responsible for the laboratory measurements. LB provided statistical expertise and developed the web-based electronic case report form. All authors contributed to refinement of the study protocol, read and approved the final manuscript.

Funding The authors have not declared a specific grant for this research from any funding agency in the public, commercial or not-for-profit sectors.

Competing interests None declared.

Patient consent for publication Not required.

Provenance and peer review Not commissioned; externally peer reviewed.

Supplemental material This content has been supplied by the author(s). It has not been vetted by BMJ Publishing Group Limited (BMJ) and may not have been peer-reviewed. Any opinions or recommendations discussed are solely those of the author(s) and are not endorsed by BMJ. BMJ disclaims all liability and responsibility arising from any reliance placed on the content. Where the content includes any translated material, BMJ does not warrant the accuracy and reliability of the translations (including but not limited to local regulations, clinical guidelines, terminology, drug names and drug dosages), and is not responsible for any error and/or omissions arising from translation and adaptation or otherwise.

Open access This is an open access article distributed in accordance with the Creative Commons Attribution Non Commercial (CC BY-NC 4.0) license, which permits others to distribute, remix, adapt, build upon this work non-commercially, and license their derivative works on different terms, provided the original work is properly cited, appropriate credit is given, any changes made indicated, and the use is non-commercial. See: http://creativecommons.org/licenses/by-nc/4.0/.

ORCID iD

Carlo Dani http://orcid.org/0000-0002-1639-621X

\section{REFERENCES}

1 Foglia EE, Jensen EA, Kirpalani H. Delivery room interventions to prevent bronchopulmonary dysplasia in extremely preterm infants. $J$ Perinatol 2017;37:1171-9.

2 Kribs A, Hummler $\mathrm{H}$. Ancillary therapies to enhance success of non-invasive modes of respiratory support - approaches to delivery room use of surfactant and caffeine? Semin Fetal Neonatal Med 2016;21:212-8.

3 Wright CJ, Polin RA, Kirpalani $\mathrm{H}$. Continuous positive airway pressure to prevent neonatal lung injury: how did we get here, and how do we improve? J Pediatr 2016;173:17-24.

4 Katheria AC, Sauberan JB, Akotia D, et al. A pilot randomized controlled trial of early versus routine caffeine in extremely premature infants. Am J Perinatol 2015;32:879-86.

5 Dekker J, Hooper SB, van Vonderen JJ, et al. Caffeine to improve breathing effort of preterm infants at birth: a randomized controlled trial. Pediatr Res 2017;82:290-6.

6 Abdel-Hady H, Nasef N, Shabaan AE, et al. Caffeine therapy in preterm infants. World J Clin Pediatr 2015;4:81-93.

7 Patel P, Mulla H, Kairamkonda V, et al. Dried blood spots and sparse sampling: a practical approach to estimating pharmacokinetic parameters of caffeine in preterm infants. Br J Clin Pharmacol 2013;75:805-13.

8 Wyckoff MH, Aziz K, Escobedo MB, et al. Part 13: neonatal resuscitation: 2015 American Heart Association guidelines update for cardiopulmonary resuscitation and emergency cardiovascular care (reprint). Pediatrics 2015;136 Suppl 2:S196-218.

9 Sandri F, Plavka R, Ancora G, et al. Prophylactic or early selective surfactant combined with nCPAP in very preterm infants. Pediatrics 2010;125:e1402-9.

10 Papile LA, Burstein J, Burstein R, et al. Incidence and evolution of subependymal and intraventricular hemorrhage: a study of infants with birth weights less than 1,500 gm. J Pediatr 1978;92:529-34.

11 de Vries LS, Eken P, Dubowitz LM. The spectrum of leukomalacia using cranial ultrasound. Behav Brain Res 1992;49:1-6.

12 Committee for the Classification of the Retinopathy of Prematurity. An international classification of infection or inflammation of retinopathy of prematurity. Arch Ophthalmol 1984;102:1130-4.

13 Bell MJ, Ternberg JL, Feigin RD, et al. Neonatal necrotizing enterocolitis. therapeutic decisions based upon clinical staging. Ann Surg 1978;187:1-12.

14 Stoll BJ, Hansen NI, Bell EF, et al. Trends in care practices, morbidity, and mortality of extremely preterm neonates, 1993-2012. JAMA 2015;314:1039-51.

15 Poets CF, Brockmann PE. Myth: gastroesophageal reflux is a pathological entity in the preterm infant. Semin Fetal Neonatal Med 2011;16:259-63.

16 Moschino L, Zivanovic S, Hartley C, et al. Caffeine in preterm infants: where are we in 2020? ERJ Open Res 2020;6:00330-2019. 